\title{
A Novel Cooperative Multicast Scheme Based on Fountain Code*
}

\author{
Qinghe Du ${ }^{1,2}$, Pinyi Ren ${ }^{1}$, Jincheng Lu' ${ }^{1}$, Zhigang Chen ${ }^{1}$ \\ ${ }^{1}$ School of Electronic and Information Engineering, Xi' an Jiaotong University, China \\ ${ }^{2}$ National Mobile Communications Research Laboratory, Southeast University, China \\ Email: duqinghe@mail.xjtu.edu.cn,pyren@mail.xjtu.edu.cn, ljc162002@stu.xjtu.edu.cn, zgchen@mail.xjtu.edu.cn
}

Received May, 2013

\begin{abstract}
Multicast is an efficient way to support emerging multimedia services over wireless network. Fountain codes are used in multicast systems to enable a robust transmission without CSI feedback and ARQ. We propose a cooperative multicast scheme based on fountain code to improve the performance of multicast. The users are coordinated with each other to decode the message at different time slots within the data transmission of a multicast session. Specifically, we take the local channel state information (CSI) and the local residual energy information (REI) into consideration, and apply a relay-selection and power-allocation strategy in our cooperative multicast scheme to prolong the network lifetime, while keeping the transmission delay as low as possible. The simulation results show that the proposed scheme can achieve a good tradeoff between transmission delay and network lifetime.
\end{abstract}

Keywords: Cooperative Multicast; Fountain Code; Residual Energy; Lifetime

\section{Introduction}

Recently, many important applications, such as video conferencing, mobile TV, software updating, etc, require the support of multicast. Multicast communication, which can simultaneously deliver data stream to many subscribers over a common channel, has become one of the main features of the next generation of mobile communication, such as IPTV over WiMax [1] and MBMS in LTE [2]. In multicast systems, data transmission only involves the downlink. A large number of multicast subscribers are usually located in different places and experience different channels fading, so their channel qualities may be profoundly different. To ensure fairness, the multicast transmission rate is determined by the user with the worst channel quality, which limits the efficiency of multicast transmission.

Another challenge the multicast system faces is how to transmit reliably when the channel conditions of the multicast group users are different and dynamically changing. Conventional fixed-rate forward error correction (FEC)

\footnotetext{
*This paper is supported by the National Natural Science Foundation of China under Grant No. 61102078, the National Science and Technology Major Project under Grant No. 2010ZX03003-004-01, the Specialized Research Fund for the Doctoral Program of Higher Education under Grant No.20110201120014, the Open Research Fund of National Mobile Communications Research Laboratory, Southeast University (No.2011D10), and the Fundamental Research Funds for the Central University.
}

code can recover some loss of packets, but it has to be designed to carry a rate below the capacity of the channel, which means the channel conditions should be known at the transmitter. In multicast system, the instantaneous channel state feedbacks for large number of users will be a huge overhead. Furthermore, for any fixed-rate FEC coding, the error or loss is still unavoidable during transmissions in time-varying wireless fading channels. When packet loss occurs, unicast transmissions can use automatic repeat request (ARQ) techniques [3] to handle it, with decreased throughput induced. However, ARQ techniques are not suitable to multicast system, since different users may lose different packets, the source has to repeat a large number of packets for different users.

Fountain codes, or rateless codes, can adapt its rate to the channel realization and require no knowledge of channel state information at the transmitter [4-5]. The transmitter can generate as many encoding symbols as needed, depending on the instantaneous quality of the channel of the receivers. The receivers keep accumulating mutual information from the source and relays, until they can successfully decoding the message. As the fountain code enables a reliable multicast transmission without CSI feedback and ARQ, it has been introduced into the standards of MBMS and DVB [6,7]. The users with different channel qualities have different speeds to accumulate the mutual information. A straightforward approach to improve the performance of multicast com- 
munication is let the users decode message first help the users decode message after. In this paper, we introduce a cooperative scheme based on fountain code to improve the performance of multicast communication, which is different from previous cooperative schemes in point-topoint scenarios $[8,9]$.

Due to user cooperation and fountain coding, we can improve the efficiency and reliability of multicast transmission. Most user terminals are battery-powered, and forwarding message for other users will consume a lot of power. Letting all decoded users forward message with maximum transmission power can obviously bring great performance gain for multicast transmission, but the shortcoming of this approach is that it is not an energy efficient way and will shorten the network lifetime, which is measured by the first node running out of its energy in the network [10]. We take the residual energy and local channel state information of users into consideration, and apply a relay selection and transmission power allocation algorithm in our cooperative multicast scheme. Simulation results show that the proposed scheme can achieve a good tradeoff between transmission delay and network lifetime.

The rest of this paper is organized as follows. The system model is introduced in Section 2. Section 3 describes the proposed cooperative multicast scheme. Simulations results are presented and discussed in Section 4 , illustrating the performance of the proposed scheme. Finally, conclusions are drawn in Section 5.

\section{System Model}

\subsection{Network Model}

Consider a wireless multicast network with a circular cell of radius as shown in Figure 1. The BS is located at the center of the cell and multicasts to $\mathrm{M}$ users. The $\mathrm{M}$ users are randomly located in the cell with uniformly distribution. The location of user $i(1 \leq i \leq M)$ can be determined by $\left(d_{i}, \theta_{i}\right)$, where $0 \leq d_{i} \leq R$ and $0 \leq \theta_{i} \leq 2 \pi$. In the paper, we consider the large-scale path loss attenuation, small-scale fading and additive white Gaussian noise (AWGN) in the wireless channel, the link between any two nodes are independent. Path-loss attenuation is determined by the geographical environment and distance between the receiver and the transmitter, so the path loss between user $i, j$ can be modeled as

$$
P L\left(r_{i, j}\right)=r_{i, j}^{-\eta}
$$

where $\eta$ is the path loss parameter, $r_{i, j}$ is the transmission distance between user $i, j$, given by

$$
\left(r_{i, j}\right)^{2}=d_{i}^{2}+d_{j}^{2}-2 d_{i} d_{j} \cos \left(\theta_{i}-\theta_{j}\right)
$$

Small-scale fading is caused by multiple versions of a transmitted signal with different delays and independent

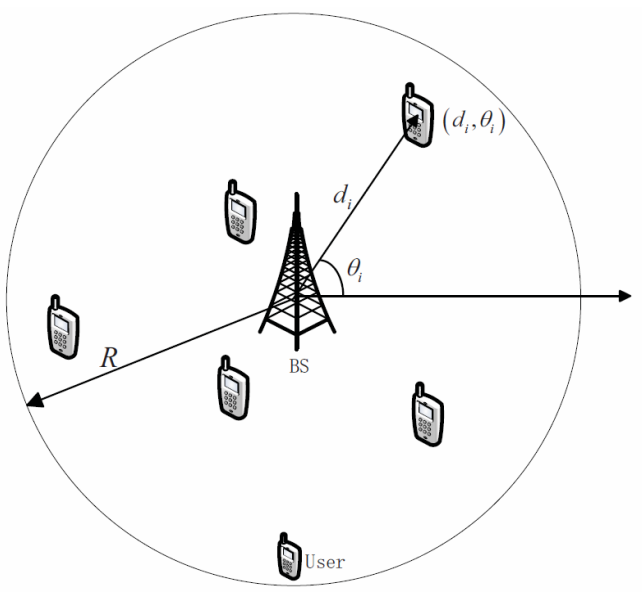

Figure 1. Network model.

of the path loss, we commonly use the Rayleigh flat fading to describe it, which can be modeled as a zero-mean circularly symmetric complex Gaussian random variable with unit variance. All users in the network are in the half duplex mode; they cannot transmit and receive on the same frequency band at the same time.

\subsection{Fountain Code}

Fountain code are applied in BS, a source file is split into $\mathrm{K}$ packets with equally size, then the encoder randomly chooses a certain number of source packets and combines them to an encoded packet by an exclusive-OR operation, then the encoded packet is broadcast to the users through wireless channel. This procedure can be repeated continuously until the BS has received the ACK signals of all multicast group users. Due to a cyclic redundancy check (CRC), the receiver knows the encoded packet is either correctly or erroneous received. If errors occur, this packet will be dropped by the receiver. A fountain code decoder tries to recover the source packets from the received encoded packets after accumulating a sufficient number of correct encoded packets, usually this sufficient number is slightly higher than $\mathrm{K}$, an additional overhead of about $5-10 \%$ of $\mathrm{K}$ is typically required [11]. As users with different conditions have different packet loss rate, they need different transmission time to receive a sufficient number of packets and decode the message.

\subsection{Cooperative Multicast Scheme}

Make different users collaborate to accomplish the data transmission is suitable for multicast scenarios based on two reasons. Firstly, users are the part of multicast group, so the security and incentive are no need to concern. Secondly, the information-theoretic results of [12] suggest that cooperation can lead to significant diversity enhancement and reduce the packet losses. In our scheme, a multicast session consists of two kinds of transmission 
modes, (i) point-to-multipoint (i.e. direct multicast), (ii) multipoint-to-multipoint (i.e. cooperative multicast). At the beginning of the multicast session, BS broadcast the encoded packets to multicast group users with a fixed transmission rate, and every user keeps accumulating mutual information from BS. It is a point-to-multipoint transmission mode as conventional multicast. For user $i$ located at $\left(d_{i}, \theta_{i}\right)$, the received signal from $\mathrm{BS}$ is given by

$$
y_{i}^{D M}=\sqrt{P_{s} r_{s, i}^{-\eta}} h_{s, i} x_{f}+n_{i}
$$

with received SNR

$$
\gamma_{i}^{D M}=\frac{P_{s}\left|h_{s, i}\right|^{2} r_{s, i}^{-\eta}}{N_{0}}
$$

where the superscript "DM" means direct multicast, $P_{s}$ is the transmission power of $\mathrm{BS}, r_{s, i}$ is the distance between BS and user $i, h_{s, i}$ is the Rayleigh flat fading, $h_{s, i} \sim C N(0,1), \quad x_{f}$ is a unit power symbol encoded by fountain code, the $n_{i}$ is the additive white Gaussian noise with a variance of $N_{0}$. If the received SNR is higher than a threshold $\gamma_{0}$, we assumed the receiver is able to decode the received message with a negligible probability of error, otherwise we assume the receiver cannot decode the packet correctly, and drop this packet. Obviously, some users with good channel conditions will receive enough correct encoded packets and decode the multicast message before other users whose channel conditions are not good. These good users have the ability to help the users that are still accumulating the mutual information from BS; we called these users candidate relays (CR). By relay selection, we choose some relays out of candidate relays to forward message; we use set $D$ to represent relays. Then users in $D$ can re-encode the packets using the same rule as the BS, and simultaneously broadcast the encoded symbols with the BS on the common channel. We assume all candidate relays involved in cooperation are synchronized and the delay spread of arriving signals is negligible, which is valid in narrow-band wireless communication. When users in D begin to forward packets, it is a multipoint-to-multipoint cooperative multicast. The users with bad channel conditions will receive message from $\mathrm{BS}$ and cooperative users, the received signal is given by

$$
y_{i}^{C M}=\left(\sqrt{P_{s} r_{s, i}^{-\eta}} h_{s, i}+\sum_{j \in D} \sqrt{P_{j} r_{i, j}^{-\eta}} h_{i, j}\right) x_{f}+n_{i}
$$

with received SNR

$$
\gamma_{i}^{C M}=\frac{\left|\sqrt{P_{s} r_{s, i}^{-\eta}} h_{s, i}+\sum_{j \in D} \sqrt{P_{j} r_{i, j}^{-\eta}} h_{i, j}\right|^{2}}{N_{0}}
$$

where the superscript "CM" means cooperative multicast, $P_{j}$ is the transmission power of relay $j$ in set $D$, the value of $P_{j}$ will be determined in power allocation section. We do not use orthogonal channels to forward message is based on the considerations of spectrum efficiency and multicast channel sharing behavior.

If all decoded users forward message with the maximum transmission power for other users have not yet decode the message, clearly, significant gain will be achieved. But as the multicast session continue, more and more users have decoded the message, let all of them forward message for only several users is a waste of energy. And some users with good channel conditions forward for others with maximum transmission power for a long time, their residual energy will be running out quickly, which is the situation we try to avoid. So we proposed a relay selection and power allocation strategy to coordinate with a higher energy efficiency and prolong the network lifetime.

\section{Relay Selection and Power Allocation Strategy}

If all decoded users forward message with the maximum transmission power for other users have not yet decode the message, clearly, significant gain will be achieved. But as the multicast session continue, more and more users have decoded the message, let all of them forward message for only several users is a waste of energy. And some users with good channel conditions forward for others with maximum transmission power for a long time, their residual energy will be running out quickly, which is the situation we try to avoid. So we proposed a relay selection and power allocation strategy to coordinate with a higher energy efficiency and prolong the network lifetime.

\subsection{Relay Selection}

In multicast scenario, there are some users that have decoded the message, but there are no other users located nearby, or users nearby have decoded the message too. If these users forward message, its cooperation gain is small. So we proposed a relay selection procedure to filter these kinds of users to improve the energy efficiency. The procedure is shown in Figure 2, contains the following steps.

1) BS encode the input packets with fountain code and broadcast encoded symbols on multicast channel, multicast group users receive the signals and accumulate correct fountain code symbols until they can decode the received message. Then these users send out an ACK signal on a feedback channel to inform the BS that it has the correctly receive the input packets and become candidate relays $(\mathrm{CR})$. 

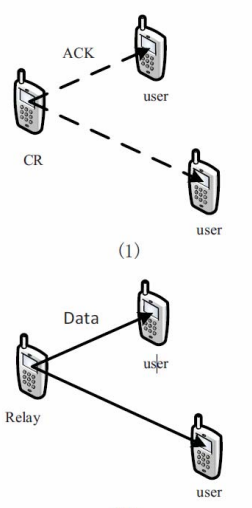

(3)

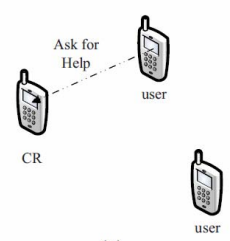

(2)

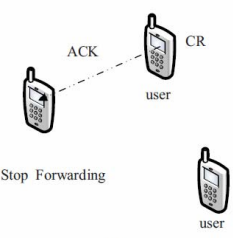

(4)
Figure 2. Relay selection.

2) The users that have not decode the message listen on the feedback channel to monitor the ACK signal. The SNR of the received ACK signals are measured and compare to a predetermined SNR threshold. If the SNR of received ACK signal is lower than the threshold, it indicates that the link conditions between the two users are not good enough to forward message. If the SNR of received ACK signal is greater than, the users will send a signal to the user who just completes the decoding for help, which includes its own ID and the ID of candidate relay.

3) If a candidate relay hear the signal ask it for help, it will recode the ID of the users who ask for help, and became a relay involve in cooperation. It begins to re-encode the decoded packets and forward them simultaneously with BS and other relays.

4) When the user completes the decoding with the help of BS and relays, it will broadcast an ACK signal too. The relays monitor the ACK signals on the feedback channel and remove the ID, if the ACK signal is from the user who asks for the relay to help before. When the relay find all users it help have decode the message, this relay will stop forwarding message and exit cooperation in this multicast session.

\subsection{Power Allocation}

Let $e_{i}[\mathrm{~m}]$ be the residual energy of user $i$ at the beginning of the $m$-th time slot, $P_{i}[m]$ is the transmission power of user ${ }^{i}$ in the period of the $m$-th time slot. So we can get $e_{i}[m+1]=e_{i}[m]-P_{i}[m] \cdot T_{s}$, and $T_{s}$ is the length of each time slot. At the beginning of a time slot, the selected user determine the transmission power by its residual energy. Assuming that there are $L$ levels of residual energy, $E=\left(\varepsilon_{l-1}<e_{i}[m] \leq \varepsilon_{L}\right)$, then the user $i$ can obtain its energy index(EI) during $m$-th time slot, $\mathrm{EI}_{i}[m]=l$, if $\varepsilon_{l-1}<e_{i}[m]<\varepsilon_{l}, \quad l \in(1, L), \quad \varepsilon_{0}=0$. Accordingly, the transmission power of users involved in cooperation can be equally divided into $L$ discrete power levels, We assume that $P_{i}[m] \in\left(P_{\min }, P_{\max }\right)$, and the interval between two levels is $\delta$,

$$
\delta=\frac{P_{\max }-P_{\min }}{L-1}
$$

When the user $i$ determine $\mathrm{EI}_{i}[\mathrm{~m}]$ by compare its residual energy to set $\mathrm{E}=\left(\varepsilon_{1}, \varepsilon_{2}, \ldots, \varepsilon_{L}\right)$, then the transmission power in $m$-th time slot is determined by

$$
P_{i}[m]=P_{\min }+\left(E_{i}[m]-1\right) \delta
$$

\section{Simulation Results}

The performance of the proposed cooperative multicast scheme is evaluated using Monte Carlo simulations through MATLAB. In our simulations, we randomly generate $\mathrm{M}$ user locations uniformly distributed inside a circle with $R=100$. The path loss parameter is $\eta=2.6$, channel gains are generated independently following the complex Gaussian distribution $C N(0,1)$ and vary independently in each time-slot.

To analyze the network lifetime performance of proposed scheme, we assume the transmission rate of multicast data stream is 4 on unit bandwidth, and then if the received SNR is higher than threshold $\gamma_{0}=15$, the users can accumulate a correct packet. And we assume if users can accumulate 1000 packets, they can decode the message. The transmission power of BS is 1, and remains unchanged during the multicast session. The transmission power range of a relay user is $(0.01,0.1)$, and there are $L=10$ power levels, so the interval of power levels is $\delta=0.01$. The initial residual energy of all user is $e_{0}$, we assume $e_{0}=100$, the length of each time-slot $T_{s}=1$, the relay selection threshold is $\gamma_{1}=15$. We also simulate the simple cooperative multicast scheme that without relay selection and power allocation, the users begin to forward the message with maximum transmission power after they have decoded the message. We set $M=100$, and varied the $P_{s} / N_{0}$ rom $64 \mathrm{~dB}$ to $70 \mathrm{~dB}$. The result is showed in Figure 3; the vertical axis is the network lifetime normalized by the maximum value in the simulations. Figure 3 shows that proposed multicast scheme perform much better than simple cooperative multicast scheme, the network lifetime is prolonged about triple times in our scheme. This gain is attributed to the relay selection and power allocation based on the local channel state information and the local residual energy information. By relay selection, the candidate relays with bad link conditions connecting to other users are not involved in the cooperation and the relays that have help the users nearby decode the message will exit cooperation too. By power allocation, the relays with abundant residual energy can support the cooperative transmission with high level of transmission power, and the relays with poor residual energy will decrease the power level to save energy. 


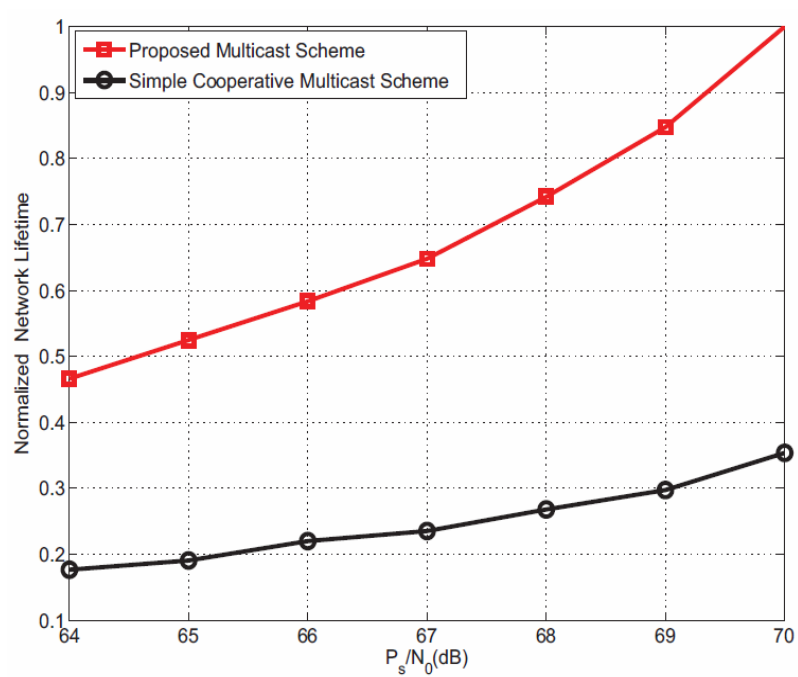

Figure 3. The performance of Network lifetime.

We also want to see the impact of the relay selection and power allocation to the multicast transmission delay performance. For comparison, the performance of the direct multicast is simulated as well as two cooperative multicast schemes under the same simulation conditions. We know the multicast transmission delay is determined by the time the worst user needs to decode the encoded packets in a multicast session. When the users are with different residual energy, the performance of transmission delay is different. At the beginning of simulation, every user have the same initial residual energy, as the multicast transmission continue, the user that running out residual energy will emerge. This procedure will last several multicast sessions; we record the multicast transmission delay of every multicast session and calculate the average transmission delay. The result is showed in Figure 4 the vertical axis is the transmission delay normalized by the maximum value in the simulations. Benefiting form cooperative diversity, the two cooperation schemes perform better than direct multicast scheme in the aspect of transmission delay. The performance gain is high especially in low SNR region, that is because some users is hard to receive correct packets without help of relays in low SNR region, and it seriously limit the performance of transmission delay. When the SNR is high, these users can receive the correct packets easier even without the help of relays, so the cooperation gain is decreased in high SNR region. Compare the two cooperative multicast schemes, we will find that the performance gap between them is relatively small. That means the relay selection and power allocation have small impact on the transmission delay in statistical average aspects. Some candidate relays are inefficient for other users, they cannot decrease too much performance gain if we do not let them involve in the cooperation.

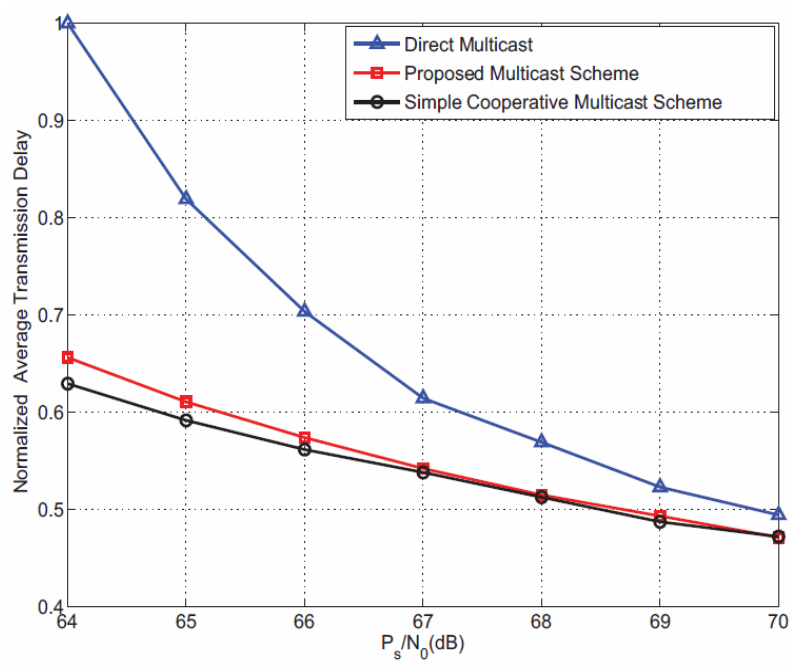

Figure 4. The performance of average transmission delay.

\section{Conclusions}

In this paper, we investigated a novel cooperative multicast scheme based on fountain code over wireless networks. By introducing fountain code, the users with different packet loss rates will decode the message at different time slots. The user who decodes the message becomes candidate relay, and decides whether to join the cooperation or not based on the link quality between itself and users. The transmission power of relays is determined by the local residual energy information. The simulation results show that our scheme can prolong network lifetime of multicast system, at the price of slight loss of average transmission delay performance.

\section{REFERENCES}

[1] J. She, P. Ho and L. Xie, "IPTV over WiMax: Key Success Factors Challenges, and Solutions," IEEE Communications Magazine, Vol. 45, No. 8, 2007, pp. 87-93. doi:10.1109/MCOM.2007.4290319

[2] S. Kota, Y. Qian, E. Hossain and R. Ganesh, "Advances in Mobile Multimedia Networking and QoS," IEEE Communications Magazine, Vol. 45, No. 8, 2007, pp. 52-53.doi:10.1109/MCOM.2007.4290314

[3] Y. Omar, M. Youssef and H. El Gamal, "ARQ Secrecy: From Theory Practice," in Proc. IEEE Information Theory Workshop ITW 2009, Oct.11-16, 2009, pp. 6-10.

[4] M. Luby, "LT Codes," in Proc. 43rd Annual IEEE Symp. Found. Computer Sci., Nov. 2002, pp. 271-280.

[5] A. Shokrollahi, "Raptor Codes on Symmetric Channels," in Proc. IEEE Int. Symp. Inform. Theory, June-July 2004, p. 38.

[6] Universal Mobile Telecommunications System (UMTS) Multimedia Broadcast Service (MBMS) Protocols and Codecs (3Gpp TS 26.346 version6.12.0 Release 6), 2008, pp. 90-105. 
[7] Digital Video Broadcasting (DVB) IP Datacast over DVB-HContent Delivery Protocols (ETSI TS 102472 v1.1.1), June 2006, pp. 57-72.

[8] J. Castura and Y. Y. Mao, "Rateless Coding for Wireless Relay Channels," IEEE Transactions on Wireless Communications, Vol. 6, No. 5, 2007, pp. 1638-1642.

[9] W. J. Lei, X. M. Li, X. Z. Xie and G. J. Li, "Wireless Cooperative Relay System Using Digital Fountain Codes," International Conference on Communications, Circuits and Systems, ICCCAS 2009.

[10] V. Shah-Mansouri and V. W. S. Wong, "Life-
time-Resource Tradeoff for Multicast Traffic in Wireless Sensor Networks," IEEE Transactions on Communications, Vol. 9, No. 6, 2010, pp. 1924-1934.

[11] R. Budde, S. Nowak and R. Kays, "Reliable Broadcast Transmission in Vehicular Networks Based on Fountain Codes," IEEE 73rd Vehicular Technology Conference (VTC Spring), 2011, pp. 1-5.

[12] A. Sendonaris, E. Erkip and B. Aazhang, "User cooperation diversity. Part I. System Description," IEEE Transactions on Communications, Vol. 51, No. 11, 2003, pp. 1927-1938.doi:10.1109/TCOMM.2003.818096 